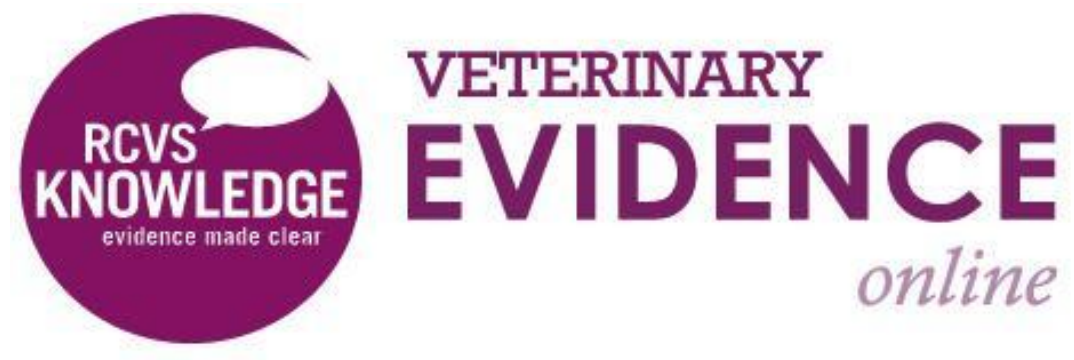

\title{
Clinical Effect of Tibial Plateau Leveling Osteotomy Compared to Lateral Fabellar Suture in Dogs
}

Wanda J Gordon-Evans DVM, PhD, DACVS, DACVSMR ${ }^{1}$

\footnotetext{
${ }^{1}$ Wisconsin Veterinary Referral Center, 360 Bluemound Road, Waukesha, WI 53188

Corresponding Author (wgordonevans@gmail.com)
}

ISSN: 2396-9776

Published: 27 July 2016

in: Vol 1, Issue 3

DOI: http://dx.doi.org/10.18849/ve.v1i3.44

Reviewed by: Kristina Kiefer (DVM, PhD) 
Objective: This manuscript used evidence based statistical methods that estimate clinical treatment effect rather than whether groups were statistically different.

Background: The previously published blinded, randomised, controlled clinical trial comparing lateral fabellar suture (LFS) and tibial plateau leveling osteotomy (TPLO) was reported with a traditional analysis comparing groups. Although this was a well-designed study, evidence based statistical methods that estimate treatment effect would be helpful to the practitioner.

Methods: The effect size and number needed to treat (NNT) were calculated for the outcome measures with significant differences between groups using the data from a previously published randomised controlled clinical trial comparing TPLO and LFS in dogs with cruciate rupture.

Results: The effect size of the peak vertical force (PVF) at a trot, 1 year after TPLO over LFS, was moderate to high (0.71) with a NNT of 6 . The NNT for satisfaction was 7.

Conclusion/Application: Based on this study, the effect size and NNTs are such that the clinical difference warrants recommending the TPLO over the LFS in large and giant breed dogs.

\section{INTRODUCTION}

The TPLO has been advocated as a better treatment for cruciate rupture in the dog based largely on a randomised controlled clinical trial published in 2013 (Gordon-Evans et al., 2013; Bergh et al., 2014). This paper describes a statistical difference in limb function between owner satisfaction and limb function (peak vertical force); however, the clinical difference between the groups using the effect size, NNT and number needed to harm (NNH) were not calculated (Gordon-Evans et al., 2013).

The effect size is a statistical measure of the treatment impact. It is the quantification of the clinical difference between treatments. Additionally, these statistics can be used to compare different studies if methodologies are similar. There are several ways to estimate effect size including Cohen's $d$ and the numbers needed to treat or harm.

Cohen's $d$ is the standardised mean difference of an outcome measure and directly describes the magnitude of the difference between the 2 groups (Thallmeier and Cook, 2015). Conventionally, Cohen's $d$ of 0.2 is small, 0.5 is medium, and 0.8 is high. The NNT is a statistic used in evidence based medicine to turn a numerical difference into a tool that can be used in clinical decision making.

The NNT is the number of patients needed to treat with the new treatment to get 1 more response than the control group. This is helpful in determining the likelihood that the treatment will be successful. The NNH is a statistic that puts complications of a treatment into a clinically relevant number describing the number of patients added to the new treatment group before a complication would be expected. The number needed to treat should be low and the number needed to harm should be high. 
The purpose of this evaluation was to provide statistics that would be useful in clinical decision making based on the previously published randomised controlled clinical trial comparing TPLO to LFS. The hypothesis was that the TPLO would have a moderate effect size with a low NNT compared to LFS.

\section{METHODS \& MATERIALS}

The randomised controlled clinical trial comparing TPLO to LFS study by Gordon-Evans was reviewed (GordonEvans et al., 2013). Although multiple outcome measures were used in the study, limb function as measured by PVF and owner satisfaction were the only measures with statistical difference. The limb function data was chosen to calculate the Cohen's d effect size at the 12 month time point (Thalheimer and Cook, 2015). The LFS was considered the control group for these calculations.

To calculate the NNTs success and failure must be defined and the dogs categorised. For PVF at the walk and trot, success was defined as PVF greater than $35 \%$ of bodyweight at a walk and $58 \%$ at a trot (Evans et al., 2005; Millis and Levine, 2014). The owner satisfaction score was on a scale of 1 to 10 with 10 being the best possible outcome. A 9 or 10 score was considered success and 8 and below to be failure. NNH was not calculated since there was no difference in complications between the 2 surgical interventions.

\section{RESULTS}

The results are presented in Table 1:

Table 1. Results of effect size and number needed to treat (NNT) calculations for Peak Vertical Forces (PVF) at both a walk and trot.

\section{Effect Size}

(Cohen's d) NNT

\begin{tabular}{lll}
\cline { 2 - 3 } PVF Walk & 0.51 & 7 \\
PVF Trot & 0.71 & 6 \\
Owner Satisfaction & & 7
\end{tabular}

\section{DISCUSSION}

For all 3 outcome measures, the treatment effect of TPLO over LFS is moderate to high.

An effect size of 0.51 is considered moderate and 0.71 would be considered moderate to high. For frame of reference, in a human metanalysis of surgical vs conservative treatment for decreasing the pain of osteoarthritis of the knee, showed an effect size of 0.35 at 3 months and 0.18 by six months. This was considered low at 3 months and negligible at 6 months (Thorland et al., 2015). In contrast, diclofenac (a nonsteroidal anti-inflammatory) showed an effect size of 0.57 for decreasing pain over placebo in adults with 
knee ostoearthritis (daCosta et al., 2016). The authors used 0.37 as the cutoff for significant clinical effect (daCosta et al., 2016).

The NNTs calculated were also clinically significant. The NNT of topical ketoprofen (a nonsteroidal antiinflammatory) for knee osteoparthritis pain relief is 7 over placebo in a recent Chochran review (Derry et al., 2016). This essentially means that 7 patients were treated in the ketoprofen group before 1 more person benefitted over placebo treatment. In contrast, in a study comparing 2 methods of preparation prior to total knee replacement the NNT was 145 for the experimental method. In other words 145 patients were prepped in the experimental treatment before 1 extra patient benefited over the standard (Lamplot et al., 2015). This helps the clinician judge the clinical utility of a new procedure, drug, or implant.

A treatment may work well but if it has a high complication rate and thus a low $\mathrm{NNH}$, it may not be worth recommending. This is valuable to note from a clinical recommendation perspective. The NNH could not be calculated from this study because the complication rates between the groups were not different.

Based on this study, the effect size and NNT are such that the clinical difference warrants recommending the TPLO over the LFS in large and giant breeds.

\section{CONFLICT OF INTEREST}

Acknowledgments: Thank you to Richard Evans for guidance in calculating the effect size and numbers needed to treat.

Funding: Funding for the original randomised controlled clinical trial was provided by Morris Animal Foundation; however, no additional funding was used for this manuscript.

Competing interests: There are no competing interests.

\section{REFERENCES}

1. Bergh, M.S. et al. (2014) Systematic Review of Surgical Treatments for Cranial Cruciate Ligament Disease in Dogs. Journal of the American Animal Hospital Association, 50 (5), pp. 315-

21.http://dx.doi.org/10.5326/JAAHA-MS-6356

2. da Costa, B.R. et al. (2016) Effectiveness of Non-Steroidal Anti-Inflammatory Drugs for the Treatment of Pain in Knee and Hip Osteoarthritis: A Network Meta-Analysis. The Lancet, 387 (10033), p. 2093.http://dx.doi.org/10.1016/S0140-6736(16)30002-2

3. Derry, S. et al. (2016) Topical NSAIDs for Chronic Musculoskeletal Pain in Adults. Cochrane Database of Systematic Reviews, [Epub ahead of print]. http://dx.doi.org/10.1002/14651858.CD007400.pub3

4. Evans, R. Horstman, C. and Conzemius, M. (2005) Accuracy and Optimization of Force Platform Gait Analysis in Labradors with Cranial Cruciate Disease Evaluated at a Walking Gait. Veterinary Surgery, 34 (5), pp. 445-9. http://dx.doi.org/10.1111/i.1532-950X.2005.00067.x

5. Gordon-Evans, W.J. et al. (2013) Comparison of Lateral Fabellar Suture and Tibial Plateau Leveling Osteotomy Techniques for Treatment of Dogs with Cranial Cruciate Ligament Disease. Journal of the American Veterinary Medical Association, 243 (5), pp. http://dx.doi.org/10.2460/javma.243.5.675

6. Lamplot, J.D. et al. (2015) Modified Protocol Decreases Surgical Site Infections after Total Knee Arthroplasty. Journal of Knee Surgery, 28 (5), pp. 395-403. http://dx.doi.org/10.1055/s-0035-1544974 
7. Millis, D.L. and Levine, D. (2014) Canine Rehabilitation and Physical Therapy. Philadelphia, PA. Saunders.

8. Thallheimer, W. and Cook, S. (2002) How to calculate effect sizes from published research: A simplified methodology. [Work-Learning Research] [Online] Available

from:http://www.bwgriffin.com/gsu/courses/edur9131/content/Effect Sizes pdf5.pdf [Accessed 25 April 2016]

9. Thorlund, J.B. et al. (2015) Arthroscopic Surgery for Degenerative Knee: Systematic Review and MetaAnalysis of Benefits and Harms. British Journal of Sports Medicine, 49 (19), pp. 1229-

35.http://dx.doi.org/10.1136/bjsports-2015-h2747rep 


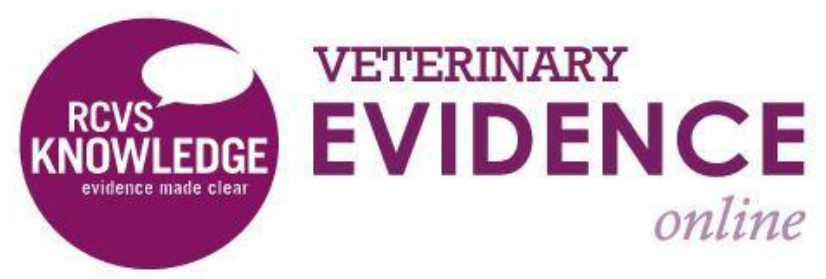

\section{Intellectual Property Rights}

Authors of Articles submitted to RCVS Knowledge for publication will retain copyright in their work, but will be required to grant to RCVS Knowledge an exclusive license of the rights of copyright in the materials including but not limited to the right to publish, re-publish, transmit, sell, distribute and otherwise use the materials in all languages and all media throughout the world, and to license or permit others to do so.

Authors will be required to complete a license for publication form, and will in return retain certain rights as detailed on the form.

Veterinary Evidence and EBVM Network are RCVS Knowledge initiatives. For more information please contact us at editor@veterinaryevidence.org.

RCVS Knowledge is the independent charity associated with the Royal College of Veterinary Surgeons (RCVS). Our ambition is to become a global intermediary for evidence based veterinary knowledge by providing access to information that is of immediate value to practicing veterinary professionals and directly contributes to evidence based clinical decision-making.

www.veterinaryevidence.org

RCVS Knowledge is a registered Charity No. 230886. Registered as a Company limited by guarantee in England and Wales No. 598443.

Registered Office:

Belgravia House

62-64 Horseferry Road

London SW1P 2AF

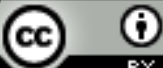

This work is licensed under a Creative Commons Attribution 4.0 International License. 